\title{
ON A CONJECTURE OF ANDREWS
}

\author{
Marie Jameson and Robert J. Lemke Oliver
}

\begin{abstract}
Following G.E. Andrews, let $q_{d}^{*}(n)$ (resp. $Q_{d}^{*}(n)$ ) be the number of partitions of $n$ into $d$-distinct parts with difference at least $2 d$ between multiples of $d$ (resp. into parts which are $\pm 1, \pm(d+2)(\bmod 4 d))$. Andrews conjectured that $q_{d}^{*}(n)-Q_{d}^{*}(n) \geq 0$ for all $n$. We prove that this conjecture is true for sufficiently large $n$ by establishing that $\lim _{n \rightarrow \infty}\left(q_{d}^{*}(n)-Q_{d}^{*}(n)\right)=+\infty$.
\end{abstract}

\section{Introduction}

The first Göllnitz-Gordon identity states that the number of partitions of $n$ into 2-distinct parts, with difference at least 4 between even parts, equals the number of partitions of $n$ into parts congruent to $\pm 1,4(\bmod 8)$. Here, a $d$-distinct partition is defined to be a partition in which the difference between any two parts is at least $d$. In addition, an identity of Schur states that the number of partitions of $n$ into 3 -distinct parts, with difference at least 6 between multiples of 3 , equals the number of partitions of $n$ into parts congruent to $\pm 1(\bmod 6)$.

It is natural to investigate whether this phenomenon has a generalization to further $d \geq 3$, and in this direction H.L. Alder [1] showed that if $d>3$, the number of partitions of $n$ into $d$-distinct parts where parts divisible by $d$ differ by at least $2 d$ is not equal to the number of partitions of $n$ into parts taken from any set of integers whatsoever. G.E. Andrews considered a different generalization by considering the functions

$$
\begin{aligned}
q_{d}^{*}(n) & :=p(n \mid d \text {-distinct parts, no consecutive multiples of } d) \\
Q_{d}^{*}(n) & :=p(n \mid \text { parts } \equiv \pm 1, \pm(d+2)(\bmod 4 d)) .
\end{aligned}
$$

At a 2009 conference in Ottawa, he made the following conjecture ${ }^{1}$ to accompany Alder's Conjecture (for more information on Alder's Conjecture, see [2], [3], [4], [7], and $[8])$.

Conjecture (Andrews). For $d>1$ and $n \geq 1$, we have that

$$
q_{d}^{*}(n)-Q_{d}^{*}(n) \geq 0 .
$$

Clearly, the conjecture holds for $d=2$ and $d=3$ by the Göllnitz-Gordon and Schur identities. Although the truth of this conjecture remains open, we show that the conjecture holds for sufficiently large $n$.

\footnotetext{
Received by the editors July 7,2009 .

${ }^{1} \mathrm{~A}$ few days after the conference, in a private communication, he modified the conjecture. We are concerned with this modification.
} 
Theorem 1.1. For fixed $d>3$,

$$
\lim _{n \rightarrow \infty}\left(q_{d}^{*}(n)-Q_{d}^{*}(n)\right)=+\infty .
$$

In addition to making the above conjecture, Andrews defined

$$
Q_{d}^{* *}(n):=p(n \mid \text { parts } \equiv \pm 1, \pm(d+2), \pm(d+6), \ldots, \pm(d+4 j+2)(\bmod 4 d)),
$$

where $j=\lfloor(d-2) / 4\rfloor$, and wondered which values of $d$ would yield

$$
q_{d}^{*}(n)-Q_{d}^{* *}(n) \geq 0 .
$$

Clearly, (1.3) implies the truth of Andrews's conjecture. Unfortunately, it is not true for all values of $d$ (it fails, for example, when $d=14$ and $n=644$ ). We find which values of $d$ cause (1.3) to hold (or fail) asymptotically.

Theorem 1.2. Assuming the notation above, the following are true:

(1) If $4 \leq d \leq 13$ or $d=17$, and $d \neq 6$ or 10 , then

$$
\lim _{n \rightarrow \infty}\left(q_{d}^{*}(n)-Q_{d}^{* *}(n)\right)=+\infty .
$$

(2) If $d=14$ or 15 , or $d \geq 18$, then

$$
\lim _{n \rightarrow \infty}\left(Q_{d}^{* *}(n)-q_{d}^{*}(n)\right)=+\infty .
$$

Remark. To establish Theorem 1.2, we show that the orders of $q_{d}^{*}(n)$ and $Q_{d}^{* *}(n)$ are different.

Remark. Theorem 1.2 does not apply when $d=6,10$, or 16 . In these cases, we expect $q_{d}^{*}(n)$ to be asymptotically larger than $Q_{d}^{* *}(n)$.

In the next section, to establish these results, we find an asymptotic expression for $q_{d}^{*}(n)$ by relating it to $q_{d}(n)$, where

$$
q_{d}(n):=p(n \mid d \text {-distinct parts }) .
$$

Asymptotics for $q_{d}(n)$ are known, and they have been very helpful in proving Alder's Conjecture and its refinement by Andrews. We use these formulae to prove Theorem 1.1 in Section 2.1 and Theorem 1.2 in Section 2.2.

\section{Proof of results}

As stated above, rather than find an asymptotic formula for $q_{d}^{*}(n)$ directly, we instead compare it to the function $q_{d}(n)$ without the added difference condition between multiples of $d$. We speculate that relatively few $d$-distinct partitions of $n$ have consecutive multiples of $d$, so we expect that $q_{d}^{*}(n) \asymp q_{d}(n)$. At present, this sort of relation is difficult to obtain. However, we note that

$$
q_{d}^{*}(n) \geq q_{d+1}(n)
$$

for all $d \geq 2$ and $n \geq 1$. From either G. Meinardus [5] or the authors and C. Alfes [2], we have the asymptotic formula

$$
q_{d}(n) \sim c_{0} n^{-\frac{3}{4}} \exp \left(2 \sqrt{A_{d} n}\right),
$$


where $c_{0}$ is an explicit constant depending only on $d$,

$$
A_{d}:=\frac{d \log ^{2} \rho}{2}+\sum_{r=1}^{\infty} \frac{\rho^{r d}}{r^{2}}
$$

and $\rho=\rho_{d}$ is the unique root of $x^{d}+x-1=0$ in the interval $[0,1]$. Hence, (2.1) and (2.2) imply that

$$
q_{d}^{*}(n) \gg n^{-\frac{3}{4}} \exp \left(2 \sqrt{A_{d+1} n}\right) .
$$

2.1. Proof of Theorem 1.1. We must consider $Q_{d}^{*}(n)$. A result of V.V. Subrahmanyasastri (Theorem 10, [6]) yields the following asymptotic formula for $Q_{d}^{*}(n)$ :

$$
Q_{d}^{*}(n) \sim c_{1} n^{-\frac{3}{4}} \exp \left(\pi \sqrt{\frac{2 n}{3 d}}\right),
$$

where $c_{1}$ is an explicit constant. Recalling (2.4), our task is now to show that for all $d \geq 4$,

or, equivalently, that

$$
\pi \sqrt{\frac{2}{3 d}}<2 \sqrt{A_{d+1}}
$$

$$
\sqrt{2 d A_{d+1}}>\frac{\pi}{\sqrt{3}}
$$

From (2.3), we have that

$$
2 d A_{d+1} \geq d^{2} \log ^{2} \rho_{d+1},
$$

and so we consider when

$$
\left|d \log \rho_{d+1}\right|>\frac{\pi}{\sqrt{3}} .
$$

Since $\left|d \log \rho_{d+1}\right|$ is increasing in $d$, one can verify that (2.6) holds for $d \geq 13$. A numerical computation verifies (2.5) in the remaining $4 \leq d \leq 12$.

2.2. Proof of Theorem 1.2. As in Section 2.1, Theorem 10 of [6] applies. In particular, we have that

$$
Q_{d}^{* *}(n) \sim c_{2} n^{-\frac{3}{4}} \exp \left(\pi \sqrt{\frac{n\left\lfloor 2+\frac{d-2}{4}\right\rfloor}{3 d}}\right)
$$

for an explicit constant $c_{2}$. A numerical computation now shows that

$$
\pi \sqrt{\frac{\left\lfloor 2+\frac{d-2}{4}\right\rfloor}{3 d}}<2 \sqrt{A_{d+1}}
$$

for $4 \leq d \leq 13, d \neq 6,10$, and for $d=17$. Hence, for these $d,(2.4)$ implies that

$$
\lim _{n \rightarrow \infty}\left(q_{d}^{*}(n)-Q_{d}^{* *}(n)\right)=+\infty .
$$

For the other values of $d \neq 6,10$, or 16 , instead of showing that

$$
\lim _{n \rightarrow \infty}\left(Q_{d}^{* *}(n)-q_{d}^{*}(n)\right)=+\infty,
$$


we show the stronger statement that

$$
\lim _{n \rightarrow \infty}\left(Q_{d}^{* *}(n)-q_{d}(n)\right)=+\infty .
$$

Hence, we must show that for these $d$,

$$
\pi \sqrt{\frac{\left\lfloor 2+\frac{d-2}{4}\right\rfloor}{3 d}}>2 \sqrt{A_{d}} .
$$

But

for all $d$ and

$$
\pi \sqrt{\frac{\left\lfloor 2+\frac{d-2}{4}\right\rfloor}{3 d}}>\frac{\pi}{\sqrt{12}}
$$

$$
2 \sqrt{A_{d}}<\frac{\pi}{\sqrt{12}}
$$

for $d \geq 26$. A numerical computation verifies (2.7) in the remaining cases.

Remark. One can check that (2.7) fails to hold when $d=6,10$, or 16 . Our above speculation that $q_{d}^{*}(n) \asymp q_{d}(n)$ suggests that, for these values of $d$,

$$
\lim _{n \rightarrow \infty}\left(q_{d}^{*}(n)-Q_{d}^{* *}(n)\right)=+\infty,
$$

although a stronger result along the lines of (2.4) would be needed to prove this.

\section{Acknowledgements}

The authors would like to thank George Andrews for making this conjecture. They would also like to thank Ken Ono for his many helpful comments on this paper.

\section{References}

[1] H. L. Alder, The nonexistence of certain identities in the theory of partitions and compositions, Bull. Amer. Math. Soc. 54 (1948) 712-722.

[2] C. Alfes, M. Jameson, and R. Lemke Oliver, Proof of the Alder-Andrews conjecture, Submitted for publication.

[3] G. E. Andrews, On a partition problem of H. L. Alder, Pacific J. Math. 36 (1971) 279-284.

[4] G. E. Andrews and K. Eriksson, Integer partitions, Cambridge University Press, Cambridge (2004), ISBN 0-521-84118-6; 0-521-60090-1.

[5] G. Meinardus, Über Partitionen mit Differenzenbedingungen, Math. Z. 61 (1954) 289-302.

[6] V. V. Subrahmanyasastri, Partitions with congruence conditions, J. Indian Math. Soc. (N.S.) 36 (1972) 177-194 (1973).

[7] A. J. Yee, Partitions with difference conditions and Alder's conjecture, Proc. Natl. Acad. Sci. USA 101 (2004), no. 47, 16417-16418 (electronic).

[8] — Alder's conjecture, J. Reine Angew. Math. 616 (2008) 67-88.

Department of Mathematics and Computer Science, Emory University, 400 Dowman Drive, Atlanta, GA 30322

E-mail address: mjames7@emory.edu

Department of Mathematics and Computer Science, Emory University, 400 Dowman Drive, Atlanta, GA 30322

E-mail address: rlemkeo@emory.edu 